\title{
Le langage silencieux dans les postures d'autorité
}

Isabelle Berlemont

\section{OpenEdition \\ Journals}

Édition électronique

URL : https://journals.openedition.org/communicationorganisation/2457

DOI : 10.4000/communicationorganisation.2457

ISSN : $1775-3546$

Éditeur

Presses universitaires de Bordeaux

Édition imprimée

Date de publication : 1 novembre 2000

ISSN : 1168-5549

Référence électronique

Isabelle Berlemont, «Le langage silencieux dans les postures d'autorité », Communication et organisation [En ligne], 18 | 2000, mis en ligne le 27 mars 2012, consulté le 05 août 2021. URL : http:// journals.openedition.org/communicationorganisation/2457 ; DOI : https://doi.org/10.4000/ communicationorganisation.2457

Ce document a été généré automatiquement le 5 août 2021.

(C) Presses universitaires de Bordeaux 


\title{
Le langage silencieux dans les postures d'autorité
}

\author{
Isabelle Berlemont
}

1 Le langage silencieux constitue la face cachée de la communication humaine en apparence dominée par le verbe. Campé au cœur de l'expérience professionnelle le non-verbal interpelle fortement le chercheur en communication. Mais comment rendre compte de cette terra incognita, de ce soubassement discret voire hyper-implicite de la culture sans tomber dans le délire d'interprétation? Pourtant il importe de mieux connaître le non-verbal tant il structure notre vie quotidienne et professionnelle. Le message postural ${ }^{1}$ dont il va être question ici représente un aspect bien particulier $d u$ langage silencieux. Nous envisagerons la fonction de la posture dans les domaines militaire, pédagogique, médical, sacerdotal et accessoirement juridique. Autant de «disciplines", au sens foucaldien, sigillées par l'autorité. Nous privilégierons la dimension globale de la posture, même si nous n'ignorons pas qu'elle est sous ponctuée par des kinèmes ${ }^{2}$. Nous proposerons une typologie sommaire de la gamme des postures professionnelles considérées établie d'après nos observations et la littérature existante. A partir de cette approche descriptive du postural nous aborderons sa résonance sociale et la liaison entre l'ordre gestuel et le dispositif de pouvoir.

\section{Autorité et dispositif de pouvoir}

Qu'elle soit d'essence spirituelle, sociale, scientifique ou technique, l'autorité apporte une tonalité bien particulière aux relations humaines ${ }^{3}$. L'exercice de l'autorité suppose que l'émetteur prenne un ascendant passager ou durable sur autrui. Consciente ou inconsciente, imposée ou librement consentie, cette domination est un élément concret $\mathrm{du}$ dispositif de pouvoir, au sens foucaldien du terme :

«cet ensemble résolument hétérogène, comportant des décisions réglementaires, des lois, des mesures administratives, des énoncés scientifiques, des propositions philosophiques, morales, philanthropiques, bref : du dit, aussi bien que du non-dit ${ }^{4}$ 
3 L'autorité n'est pas un phénomène isolé. Selon nous, toute posture d'autorité procède d'une relation explicite ou implicite au dispositif de pouvoir. Il existe une véritable formation discursive de l'autorité qui contient non seulement des énoncés verbaux comme "j'ordonne que vous obéissiez », mais aussi toute une gamme de postures exprimant la domination ou tout au moins la détention d'un pouvoir sur autrui. Ainsi, les communications non-verbales de l'officier, du professeur, du thérapeute, du juge et du prêtre reflètent l'autorité qui leur est conférée. Chacun de ces professionnels, au demeurant, privé des attributs et des postures spécifiques de son état perdrait sa part symbolique d'autorité aux yeux des autres. Le langage silencieux de l'autorité tout comme son registre verbal n'est pas neutre. Il s'inscrit dans un fort préconstruit, dans la formation discursive silencieuse du pouvoir et de la domination: un répertoire spécialisé qui prend aussi sa source dans l'enfance et le règne animal. Chaque signal d'autorité perçu active peu ou prou la relation du sujet à l'autorité et s'inscrit dans une relation intertextuelle avec le macrotexte du langage du pouvoir ${ }^{5}$.

\section{Formes et fonctions des postures d'autorité}

4 L'expression silencieuse de l'autorité institutionnelle est fortement ritualisée. Son «locuteur " n'est que l'interprète plus ou moins doué de la culture posturale de sa configuration $^{6}$. Ce code gestuel peut être lié au protocole spécifique régissant le langage silencieux d'un micro-pouvoir donné. Il peut aussi correspondre à des universaux transculturels : s'incliner en signe de déférence ou pointer l'index vers un subordonné pour le commander. L'utilisation du langage silencieux dans les postures d'autorité est un processus à double effet qui vise à renforcer la puissance symbolique de celui qui détient le pouvoir et à clarifier son message. Les postures de domination et de soumission ont été soigneusement étudiées par la psychologie animale et humaine. Schenkel estime que le rapport domination/soumission chez les canidae est un rite majeur d'intégration sociale : « la soumission est une contribution faite par un inférieur à l'intégration sociale harmonieuse sur la base de la différenciation sociale hiérarchique $»^{7}$. Mais le comportement des canidae n'est pas un modèle universel chez les mammifères. Ainsi, les observations de A.A. Chance ${ }^{8}$ nous montrent l'existence de comportements sociaux différenciés selon les races chez les primates. Chez les gorilles et les chimpanzés le rituel de contact prime sur celui d'agression et le postural dominant/dominé n'est pas omniprésent comme chez les babouins. Chez l'enfant, à côté du couple antagoniste dominant agressif/dominé, il existe un type de caractère très utile pour structurer la société enfantine : celle du leader, véritable médiateur qui détourne en quelque sorte l'attention du dominant agressif pour le faire participer. Face à une agression, le leader changera la tonalité de l'échange en adoptant une posture de détournement du conflit :

«il balance le haut du corps en pointant l'index dans une direction quelconque (détournant ainsi l'attention du premier), en montrant le jouet avec lequel il joue, et cela en souriant et en parlant. Le comportement du dominant agressif consiste alors généralement en une imitation du leader "... « il se met donc à l'écoute du comportement et des paroles du leader et s'engage avec celui-ci dans une série d'interactions non agressives qui débouchent souvent sur la coopération et la création d'activités nouvelles'.

On retrouve ces deux types fondamentaux de domination sur autrui : l'agression et la persuasion chez l'adulte. De sorte que les postures d'autorité seront plus ou moins 
marquées selon le caractère de l'émetteur en position d'autorité, fortement déclinées chez les dominants ou suggérées comme une convention chez les médiateurs. La posture classique de domination: tête en extension, poitrine en avant, une jambe fléchie en avant, éventuellement mains sur les hanches pourra donc selon les sujets et le contexte de la relation être pure, tronquée, limitée au port de tête et/ou à la fixité du tronc.

\section{Quelques postures professionnelles d'autorité}

6 L'examen du répertoire postural lié aux fonctions d'autorité suivantes: officier, professeur, juge, psychiatre et prêtre va nous permettre d'observer le rôle de la posture dans la relation d'autorité.

\section{L'officier ou l'emprise de l'élégance}

$7 \quad$ II existe toute une littérature et des manuels décrivant le comportement attendu de l'officier. Dans les représentations collectives, à la fois aîné protecteur, maître et garant en dernier ressort de la vie de ses hommes, il leur impose le respect par sa prestance, son élégance et sa maîtrise de la langue. Des générations de Saint-Cyriens ont mené de pair l'apprentissage des armes et des cérémonies mondaines. Les codes militaires internationaux se rejoignent pour donner de l'officier l'image d'un praticien spécialiste de la culture de l'énergie et de la souplesse ${ }^{10}$. La culture kinémorphique dominante de l'officier, telle que reflétée par les manuels militaires est travaillée par un souci esthétique manifeste. L'officier est souvent en représentation et son statut lui interdit toute outrance gestuelle qui viendrait brouiller son image. Sa formation sportive lui épargne néanmoins, hormis les cérémonies protocolaires, un maintien trop guindé. La référence implicite des manuels de formation de l'officier semble celle des canons du maintien aristocratique ${ }^{11}$ Parmi les témoignages que nous avons consulté deux éléments ressortent nettement sur la particularité du langage silencieux de l'officier. Il est d'abord le miroir idéal qui renvoie à ses hommes une image idéalisée de leur propre gestuelle. Un autre élément : l'officier établit le contact avec ses subordonnés par le regard dans les yeux ${ }^{12}$, chose qu'il partage avec d'autres professions d'autorité, mais ce regard ne cherche pas tant à sonder autrui qu'à établir un contact direct pour susciter un processus d'identification. Ce regard ne veut rien faire avouer, il veut seulement amener le récepteur à penser : cet homme m'impressionne et je vais m'efforcer de lui ressembler.

\section{Trois postures du pédagogue}

8 Les traités de pédagogie et le Code Soleil, ancien bréviaire des instituteurs, abordent la gestuelle d'accompagnement de l'enseignant soucieux d'asseoir son autorité. Ils soulignent les qualités psychologiques attendues; calme et sang froid, de manière à ne pas susciter des réactions imprévisibles chez l'auditoire. Rien ou peu sur les situations limites voire extrêmes que peuvent affronter certains enseignants dans les classes difficiles. Nous distinguons trois grandes variétés dans les postures des enseignants. Il y a le régime dominateur, médiateur et mimétique. Le premier est proposé par le type de sujet autoritaire défini dans la caractérologie reichienne comme se protégeant d'autrui 
par la stratification du cuirassement (panzerschichtung). Le tableau tonique est dominé par la raideur musculaire qui dénonce l'hyper-protection dont s'entoure le maître autoritaire. Lorsqu'il parle il parait menacer ; son buste est dressé en avant, son avant bras est projeté avec souvent le doigt tendu. Son regard ne se fixe dans les yeux d'un élève que pour y lire une marque de soumission. Cependant, si d'ordinaire la voix forte accompagnée de mimiques exprimant la colère, de froncements de sourcils, de gestes brusques, de variations rapides de postures, annonciateurs d'un possible passage à l'acte suffisent à calmer le jeu, il peut arriver que la classe soit ingouvernable. Se sentant mis en cause dans son autorité, il change de posture :

«Il crie, parfois même il se laisse aller à injurier les enfants, et il présente le spectacle, non d'un homme qui se possède, mais d'un aliéné pris d'un accès de folie furieuse ; «... «bien des maîtres sont de mauvais maîtres parce qu 'ils sont mal équilibrés » ${ }^{13}$.

$9 \mathrm{Au}$ fond l'autoritaire, irritable est dans la position du chef nommé non reconnu d'emblée par le groupe. La communication pédagogique médiatrice, elle, est riche en postures d'apaisement, d'écoute et de dialogue. Le médiateur dirige sa classe avec une autorité souple reflétée dans sa posture détendue. Le maître médiateur s'interdit toute production de posture de domination agressive. Cette qualité, condition sine qua non d'une discipline consentie est notée très tôt dans les traités pédagogiques :

«Dès qu 'on entre dans une classe on sent, etnulle erreur n 'est possible, si le maître est une volonté calme etsaine ou non. c'est-à-dire s'il est un éducateur ou non. Le premier signe extérieur de la force de la volonté c 'est le calme, c'est-à-dire la possession de soi. $»^{14}$

10 Le profil postural du médiateur est relâché sans excès, souple avec une tendance à ouvrir les mains vers l'auditoire. Il privilégie l'explication. Si le torse est à l'occasion projeté en avant, c'est dans un élan de conviction. Ses yeux se fixent dans les yeux des élèves pour y lire des marques de compréhension. Ici, c'est la conduite du dialogue qui est la source de l'autorité. L'enseignant est dans le rôle du chef naturel coopté. L'idéologie de communication mimétique avec les élèves place l'enseignant dans un paradoxe logique. Celui de l'autorité qui veut s'affirmer en se niant. II espère tirer son autorité du groupe en calquant sur lui ses attitudes. C'est le personnage caricatural du " prof copain » qui semble naturellement partager les cultures et les modes de jeunes et leurs attitudes. Cette optique démagogique ne comporte pas que des risques. Mais le maintien de la relation d'autorité demeure fragile. L'enseignant a tendance à adopter des postures labiles qui varient selon ses interlocuteurs. Ses postures ne sont que le produit des messages posturaux des jeunes qu'il copie. A l'inverse de l'officier-miroir émetteur de postures exemplaires il réfléchit en s'y conformant les postures du groupe qu'il doit encadrer.

\section{Le thérapeute ou la posture rationnelle normative}

D'ordinaire très au fait du langage du corps, car sa formation médicale inclut la symptomatologie posturale, le thérapeute fait un usage bien tempéré du verbe comme des postures. Ce système postural appauvri s'avère néanmoins efficace dans la relation analytique. Riche en signification, il contribue puissamment à asseoir le pouvoir du thérapeute sur son patient. Les principes freudiens d'écoute bienveillante et de non intervention expliquent en partie cette maîtrise du silence et de l'atonie musculaire dans laquelle les thérapeutes sont passés maîtres. La posture conseillée par les manuels 
n'est pas exempte de rigidité, elle favorise la distanciation durant l'entretien. Les figures de l'analyste en posture d'écoute et de l'analysé détendu sur son divan sont d'ailleurs emblématiques de la psychanalyse. Le thérapeute qui intervient dans le désordre des passions et de l'esprit doit toujours demeurer maître du jeu. Il endosse donc une véritable cuirasse qui le protège lui-même de la tentation de passer la frontière entre raison et délire. Cette attitude peu tonique comporte, comme tout autre système postural des successions modales, comme le souligne Scheflen qui a observé de ce point de vue une analyste durant un entretien ${ }^{15}$. Il s'agit de variations de postures infimes par rapport à celles qu'autorisent d'autres codes. Cette quasi immobilité rappelle au fond celle du magistrat. Le juge incarne la justice et rien d'autre. Si la truculence fortuite des affaires passées en correctionnelle l'autorise à prendre quelques libertés et à relâcher un peu la tension qui régit ses attitudes, dans les procès d'assises c'est la rigidité qui domine. Les procureurs observent une rigidité absolue lorsque la Cour s'installe ou lorsque un verdict lourd est ramené par les jurés. La gestuelle immobile du magistrat lui permet de ne rien laisser transparaître qui pourrait être interprété comme en décalage avec ses décisions. Semblable au garçon de café de l'Etre et le Néant, le juge est un être qui dit non, il en rajoute, en termes sartriens, par une gestuelle appropriée.

\section{Les postures de l'autorité ecclésiastique}

Dans le christianisme la gestuelle du prêtre est fixée par une tradition dont la symbolique remonte à la fondation de l'Eglise. Ainsi, dans la symbolique romane, les postures sont très codifiées. Les yeux grands ouverts regardant de face montrent la majesté et l'attention universelle, imposer la main au dessus de la tête la transmission d'un pouvoir, celui qui se dresse les bras levés entre en rapport avec la puissance céleste. L'index de la main pointé vers le haut désigne l'autorité souveraine. La station debout est privilégiée. La position assise est réservée à l'évêque et au prêtre dans l'absolution. Si par le passé l'Eglise a véhiculé de forts rapports d'autorité, son code postural contemporain diffère des autres en ce qu'il ne reproduit pas les rapports de domination communs. Ainsi, contrairement à la majorité des autres codes posturaux, pour lesquels baisser la tête et s'agenouiller est un signe d'infériorité, l'inclination n'a pas une signification de soumission:

«Dans la célébration liturgique, les divers ministres s'inclinent devant le président de l'assemblée non parce qu'il exerce un pouvoir quelconque, niais pour signifier que sa présidence est présence agissante du Christ ${ }^{16}$.

De même, le fait de se mettre en position de servir l'autre, notamment par le lavement des pieds au jeudi saint n'abaisse pas l'officiant :

« ce geste donne à voir qui est le Christ serviteur, ce qu 'il fait pour les hommes, et invite l'assemblée à vivre, à la suite du Christ, l'amour fraternel et le service mutuel, il donne ce faisant à voir à l'assemblée des fidèles le figure du Christ serviteur $»^{17}$

Ce n'est pas devant l'autorité du prêtre que les fidèles s'agenouillent, mais devant celle de Dieu. Le rituel d'allégeance au prêtre est inexistant, même si la kinésie de soumission du croyant paraît s'adresser à lui.

Nous nous sommes limités à l'examen de la signification symbolique des postures dans des fonctions d'autorité en distinguant des postures d'autorité miroir, dominatrice, médiatrice et transcendantale mais il existe encore d'autres figures. Certaines relations 
autoritaires de l'espace professionnel sont mixtes, avec une alternance de la détention de la posture d'autorité, comme la relation serveur-client dans un restaurant qui a été bien analysée par l'interactionnisme symbolique. Les postures dans les entretiens de recrutement nous semblent aussi un bon terrain d'étude. Par ailleurs, il faudrait aussi envisager le statut de la posture de l'autorité invisible, celle du pouvoir panoptique, par exemple. Rendre compte également des postures d'autorité collectives des groupes sociaux dominants sur les minoritaires. On pourra également établir une typologie des pratiques posturales professionnelles dominantes en les confrontant avec les manuels de gestuelle disponibles. On posera concomitamment un certain nombre de questions : quid du rapport nature-culture dans la gestuelle? Quelle est la part de "scientificité » et d'approximation dans les manuels de gestuelle»? Quels sont les obstacles qui empêchent un geste d'accompagnement ou de ponctuation d'atteindre l'effet escompté ? Peut-on rédiger une grammaire des fautes gestuelles?

Par ailleurs, comme les postures d'autorité professionnelles ne sont pas autonomes mais reflètent le dispositif de pouvoir elles sont comme lui susceptibles de se déplacer et d'évoluer selon le progrès technique et raffinement des rôles sociaux. Ainsi, les sciences humaines ont fortement travaillé les différents champs professionnels que nous avons abordé en y remodelant les rapports d'autorité et peut-être même les postures traditionnelles... En ce sens, il convient aussi d'adapter à notre actualité la définition foucaldienne des disciplines: «qui caractérisent, classifient, spécialisent,...distribuent le long d'une échelle, répartissent autour d'une norme, hiérarchisent les individus les uns par rapport aux autres, et à la limite disqualifient et invalident. ${ }^{18}$

\section{NOTES}

1. Nous empruntons à Jacques Corraze in Les communications non-verbales, Paris, PUF. 1983, p. 117, la définition suivante : «On entend par posture la position du corps, ou de ses parties par rapport à un système de repères déterminés. Il s'agit donc de l'orientation des éléments corporels. Dans les communications non-verbales, on use surtout de deux types de repères : l'orientation d'un élément du corps par rapport à un autre, ou au reste de ce même corps »... «l'orientation du corps, ou de ses parties, par rapport à d'autres corps ".

2. Ray Birdwhistell envisage la gestuelle comme un système de signification complexe doublant ou non le système verbal. Le kinè est le plus petit élément perceptible dans une gestuelle ; (ex : un froncement de sourcil). Sa répétition au sein d'une même séquence gestuelle engendre un kinème. Les kinèmes se combinent entre eux et se joignent à d'autres formes kinésiques pour former des kinémorphes et des kinémorphèmes. Les kinémorphes se combinent à leur tour dans des constructions kinémorphiques complexes. De sorte que la structure du code gestuel est comparable à la structure du discours en « son », mots, propositions, phrases.

3. Dès 1929, Wilhelm Reich. aborde longuement dans sa caractérologie lu personnalité autoritaire, définissant le chef comme "phallique narcissique ». Il est difficile d'ignorer que le caractère sexuel intervient d'une manière ou d'une autre dans la relation d'autorité. Dans la lignée ou non de la psychanalyse d'obédience reichienne, l'expérience historique du totalitarisme 
a généré nombre de recherches sur la relation autoritaire et la soumission à l'autorité dont la plus connue demeure celle de Stanley Milgram effectuée en laboratoire de 1950 à 1963.

4. Michel Foucault, Dits et écrits, Tome III, p. 299.

5. Cette extension de la notion d'intertextualité empruntée à Julia Kristeva au répertoire du langage silencieux ne nous paraît pas si abusive; on parle déjà d'intertextualité pour l'image. L'intertextualité du langage silencieux est directe, un geste peut en citer un autre ou indirecte : le geste peut être connoté par un autre support de sens. Ainsi, la transmission dans la mémoire collective des actes du langage silencieux passe aussi par l'écrit : la phrase « suivez mon panache blanc " génère une représentation kinésique durable.

6. Selon GW Hewes, «The anthropology of posture ", Scientific American, 1957, 196, pp. 123-132, le corps humain peut adopter un millier de postures. Les cultures choisissent dans cet éventail celles qui véhiculeront des significations données.

7. Voir R. Schenkel, "submission: its feature and function in the wolf and dog ", American zoologist, 1967, 7, p. 325, cité in Jacques Corraze, op.cit. p. 118.

8. Hubert Montagner, L'enfant et la communication, Paris, Stock 1998, pp. 41-42.

9. Ibid, p. 269-270.

10. Cette philosophie mêlant art et action martiale a été développée comme une mystique par l'écrivain japonais Yukio Mishima qui écrit dans Le Soleil et l'acier (1973) : "combiner l'action et l'art c'est combiner la fleur qui se flétrit et la fleur qui dure à jamais, mêler chez un individu les deux désirs les plus contradictoires de l'humanité » (p.68).

11. Le rituel d'intronisation des élèves officiers de Saint-Cyr avec sa formule: «à genoux les hommes, debout les officiers » rappelle la cérémonie d'adoubement des chevaliers.

12. «Un sujet de rang inférieur regarde plus quand il écoute que quand il parle alors qu'un sujet de rang supérieur ne regarde pas plus quand il écoute que quand il parle,.il a même une tendance à regarder davantage quand il parle que lorsqu'il écoute, c'est ce que Exline et coll. appellent « le comportement de dominance visuelle»" cité in: Jacques Corraze, Les communications nonverbales, op.cit. p. 111.

13. Jules Payot, Aux instituteurs et aux institutrices, conseils et directions pratiques, Paris, Armand Colin, 1901, pp. 32-33.

14. Ibid.

15. A.E Schlefen. "The signifiance of posture in communication systems ", in Psychiatry, 1964,27, p. 322,cité in Jacques Corraze, op. cit. p.126, note l'existence de-différents "points » (la conservation d'une posture déterminée) : «Le point 1 : tête légèrement penchée, détournée vers la droite et évitant le regard du sujet en position d'écoute. Puis un signal marqueur pour signifier la fin du point I à l'analysé ; tête dressée, signal de préparation à l'interprétation. Le point 2 : tête droite, regardant le sujet, c'est la position d'interprétation. Puis à nouveau un marqueur, tête légèrement tournée à droite et détournée du sujet pour marquer la fin de la période d'interprétation. »

16. Abécédaire de la célébration chrétienne, Paris, Les éditions de l'Atelier, 1999, pp. 70-71.

17. Ibid.

18. Michel Foucault, Surveiller et punir, Paris. Gallimard, 1975, p 224. 


\section{AUTEUR}

ISABELLE BERLEMONT

Université Rennes 2 